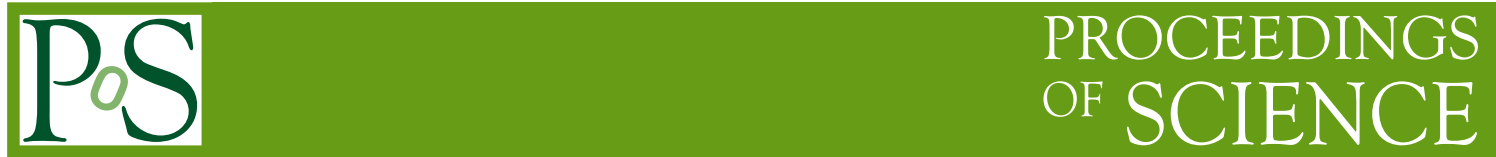

\title{
Recent Development in Proton Spin Physics
}

\section{Feng YUAN*}

Nuclear Science Division, Lawrence Berkeley National Laboratory, Berkeley, CA 94720,USA

RIKEN BNL Research Center, Building 510A, Brookhaven National Laboratory, Upton, NY

11973, USA

E-mail: fyuan@lbl.gov

In the naive model of the proton, its $\frac{1}{2}$ spin is carried by its quark constituents. However, experiments over the last several decades have shown that the quark spin only contribute a small portion of the proton spin. In this talk, I will present recent developments exploring this proton spin puzzle, and focus on the progress made in the last few years on the longitudinal spin physics, the generalized parton distribution physics, and the transverse spin physics.

LIGHT CONE 2008 Relativistic Nuclear and Particle Physics

July 7-11, 2008

Mulhouse, France

\footnotetext{
* Speaker.
} 


\section{Introduction}

Nucleon structure is fundamental in sub-atomic physics, and has been under intensive investigation in the last and the beginning of this century. For example, the experimental studies on the electro-magnetic form factors in 30s and on deep inelastic scattering in 70s have revealed nontrivial internal structure of nucleon, and the latter in particular found partons (quark and gluon) inside nucleon. These studies are still at frontier in basic science research at present. Both RHIC at BNL and CEBAF at JLab, HERMES at DESY, COMPASS at CERN, together with other experimental facilities around the world, are running important programs for the research on the nucleon structure. Most prominently the proton spin structure has been the focus of the theoretical and experimental hadronic physics in recent years.

The proton is a spin $1 / 2$ particle. The proton spin story began at 60 s when quark model was proposed. In the naive quark model, the nucleon is made of three quarks, and the proton spin came from the quark spin. Because the quark model was so success, the idea that the proton spin is entirely coming from the quark spin had been rooted in people's mind for almost three decades, until the EMC experiments in late 80s. This experiment found that the quark spin contribution to the proton spin is very small, consistent with zero [1]. This is the so-called "spin crisis". Of course, QCD, the strong interaction theory used to describe hadron property and their interactions, is a far more fruitful theory than the naive Quark Model. In the language of QCD, the nucleon does not just consist of quarks, but also of gluons, all of which have orbital motion inside the nucleon, and all of which will contribute to the proton spin. So, the ultimate goal of the spin physics is to understand the contributions that go into fulfilling the spin sum rule for the proton [2],

$$
\frac{1}{2}=\frac{1}{2} \Delta \Sigma(\mu)+\Delta g(\mu)+L_{q}(\mu)+L_{g}(\mu),
$$

where $\Delta \Sigma$ and $\Delta g$ are the total quark and gluon helicity contributions to the proton spin, respectively,

$$
\Delta \Sigma(\mu) \equiv \sum_{q} \int_{0}^{1} d x[\Delta q(x, \mu)+\Delta \bar{q}(x, \mu)], \Delta g(\mu)=\int_{0}^{1} d x \Delta g(x, \mu) .
$$

$L_{q}(\mu)$ and $L_{g}(\mu)$ are quark and gluon orbital angular momentum (OAM), respectively. The scale $\mu$ indicates the momentum scale at which these quantities are measured.

There have been tremendous experimental efforts to pin down this proton spin puzzle, including the lepton-nucleon experiments, HERMES at DESY, COMPASS at CERN, and JLab experiments; the proton-proton collider at RHIC at BNL. Besides these experiments, a number of future facilities dedicated for spin physics are also on the horizon, including the polarized electronproton colliders (EIC, eRHIC, or ELIC), the polarized fixed target $p p$ scattering at JPARC, and the polarized $p \bar{p}$ scattering at GSI-FAIR. All these experiments have their unique coverage on the studies of quark spin, gluon spin, and orbital angular momenta of quarks and gluons, and they are complementary to each other as well.

In this talk, I will try to summarize recent developments in proton spin physics. Of course, it is hard to include all the exciting progress made in the last few years. I would like to focus on the three major areas: longitudinal spin physics; generalized parton distributions (GPDs); the transverse spin physics. 


\section{Longitudinal Spin Physics}

The quark and gluon helicity distributions can be studied from the longitudinal spin program, where a longitudinally polarized lepton beam (or proton beam) scatters on a longitudinally polarized nucleon. The experiments measure the double spin asymmetry $A_{L L}$, which can be used to extract the polarized quark and gluon densities. The polarized quark distributions have been well determined from the polarized deep inelastic scattering (DIS) experiments, and it was found that the total quark spin contribution to the proton spin is about $30 \%$, see for example $[3,4]$. However, there are still two important issues about the quark helicity distribution remained to be settled: one is the quark polarization at large- $x$; one is the sea quark polarization.

Power-counting rules for the large- $x$ parton distributions were derived many years ago based on perturbative QCD combined with a $S$-wave quark model of hadrons. The basic argument is that when the valence quark carries nearly all of the longitudinal momentum of the hadron, the relevant QCD configurations in the hadronic wave function become far off-shell and can be treated in $\mathrm{pQCD}$. The leading diagrams associated with the leading Fock state of the proton wave function predict that the positive helicity (quark spin aligned with the proton spin) quark distribution $q^{+}(x)$ scales as $(1-x)^{3}$, whereas the negative helicity (quark spin anti-aligned with the proton spin) quark distribution $q^{-}(x)$ is suppressed by $(1-x)^{2}$ relative to the positive helicity one, scaling as $(1-x)^{5}$ at large $x$ [5]. The direct consequence of these power laws for the quark distributions is that the ratio of polarized quark distribution $\Delta q(x)=q^{+}(x)-q^{-}(x)$ over the unpolarized quark distribution $q(x)=q^{+}(x)+q^{-}(x)$ approaches 1 in the limit $x \rightarrow 1$; i.e., at large $x, q^{+}$dominates over $q^{-}$. When this prediction is compared to the experimental data, it is interesting to observe that, for the up quark the ratio increases with $x$, and seems to approach 1 at large $x$. However, the ratio for the down quark is still far below 1, and remains negative for a wide range of $x \leq 0.6$ [6]. In our recent study [7], we have reexamined the large- $x$ quark helicity distributions in this perturbative QCD framework, and found that for the negative helicity distribution $q^{-}$, there exist large logarithmic enhancements from the $\left|L_{z}\right|=1$ Fock states. Because of that, the $q^{-}$distribution will be dominated by the contributions from $L_{z}=1$ Fock state of the proton, and scale as $(1-x)^{5} \log ^{2}(1-x)$. With this large logarithmic modification, we can explain the discrepancy between the power-counting rule and experimental data [7]. It will be interested to see how this compares with the future data from RHIC and JLab, and helps us to learn more about the QCD dynamics associated with the large-x quark polarization.

From the inclusive measurements in the DIS experiment, it is difficult to get the information on the quark "sea" contribution to the proton spin. In recent years, there has been great interest to study the quark "sea" polarization from the so-called semi-inclusive DIS (SIDIS) experiments, where a final state hadron (for example, a Pion or Kaon) is detected. The experimental results from the HERMES collaboration have revealed nontrivial sea structure in nucleon [8]. For example, the unpolarized strange quark distribution is normally parameterized as $(\bar{u}(x)+\bar{d}(x)) / 2$. However, the HERMES results found that the strange quark distribution is quite different from this parameterization: suppression at moderate large- $x$ while enhanced at moderate small-x. The polarized strange quark distribution is also different from most model assumptions, being positive at the $x$ range of 0.05-0.2. Future SIDIS experiments at JLab and $W$ measurement at RHIC shall be able to solve this issue. The theorists should also look back their model analysis for the strange polarization in 
nucleons.

Recently, the gluon spin contribution has attracted much attention. The polarized DIS experiments can also provide the constraints on the gluon helicity distributions. However, the gluon contributes to the polarized DIS cross section at the NLO level, and we can only get the gluon information from the scaling violation of the polarized structure function. Because of the limitation of the current available experimental data, the determination of gluon helicity distribution from the DIS experiments suffers from very large uncertainties.

In the hadronic reactions at RHIC, on the other hand, gluons enter at the leading order, and the double spin asymmetry for the hard scattering processes can directly probe the polarized gluon distribution. There have been very exciting experimental results from both PHENIX and STAR collaborations on the gluon helicity distribution studies at RHIC $[9,10]$. PHENIX experiments especially studied the double spin asymmetry for the inclusive hadron $\left(\pi^{0}\right)$ production, whereas the STAR experiments focused on the inclusive jet production in the polarized proton-proton scattering. From their analysis, both experiments found that gluon polarization tends to be small $[9,10]$. The direct photon production at RHIC is also important in the future to pin down the sign of the gluon's polarization, because it depends linearly on the gluon polarization.

Theoretically, it is very important to perform a global analysis of the polarized parton distributions from fitting to the world-wide experimental data. In general, in these global analysis, one has to make some generic assumptions about the functional form (in terms of the unpolarized parton distributions) with a few parameters to fit to data. Recently, there has been a great effort to include the RHIC experimental data in the global fit [11] at the next-to-leading order of perturbative QCD. It is very challenging to perform a global fit at this order because the calculation of the hadronic processes for RHIC experiments at NLO is very costly in computing power and time. In this global fit, the authors made use of the so-called generalized Mellin transformation technique to reduce the computing loads, and they were able to perform the global fit for the first time by including hadronic data at the NLO level [11]. Their findings are consistent with the experimental analysis $[9,10]$, i.e., the gluon polarization is small, but of course with large uncertainties. Their best fit gives the following quotes for the first moment of the parton helicity distributions,

$$
\Delta \Sigma=0.243, \Delta g=-0.084, \Delta s=-0.057, \Delta \bar{u}=0.036, \Delta \bar{d}=-0.115
$$

where the uncertainty for $\Delta g$ is about 0.5 for $\Delta \chi^{2} / \chi^{2}=2 \%$ choice for the error bar calculation [11]. The future RHIC experiments shall reduce this uncertainty to a great deal with more data and statistics.

\section{Generalized Parton Distributions}

Generalized Parton Distributions were motivated by studying nuclear spin physics. They are a new type of parton distributions, which contain much more information than any individual nuclear observables that have been considered so far. They depend on three variables: $x$ the parton momentum fraction, $t$ the momentum transfer, and $\xi$ the skewness parameter. The GPDs can be reduced to the normal parton distributions in the limit of $\xi=0$, and their first moments are related to the nucleon form factors. One very important motivation to study GPDs is that they can provide 
information on the quark orbital angular momentum contribution to the proton spin [12],

$$
J_{q}=\frac{1}{2} \Sigma_{q}+L_{q}=\lim _{t \rightarrow 0} \frac{1}{2} \int d x x\left[H^{q}(x, \xi, t)+E^{q}(x, \xi, t)\right],
$$

where $J_{q}$ is the total quark contribution to the proton spin. After subtracting the helicity contribution $\Sigma_{q}$ from the polarized DIS measurements, the above equation will provide the quark orbital angular momentum contribution to the proton spin. The GPDs can be measured in many different experiments, for example, Deeply Virtual Compton scattering (DVCS) and hard exclusive meson production at HERMES, JLab, and COMPASS, and the GPD framework can link together the results from these measurements, along with the traditional PDF and form factor measurements.

Recent experiments on the DVCS from JLab Hall A [13] and HERMES at DESY [14] have shown strong sensitivity to the quark orbital angular momenta in nucleon. However, these measurements do not reveal the whole structure information of the GPDs, and the analysis has to depend on the model parameterizations which have the quark orbital angular momentum as input. Therefore, these analysis is very much model dependent. This has also been shown in the paper by the HERMES collaboration, where two different model assumptions for the GPDs lead to very different constraints for the orbital angular momenta from up and down quarks. From the theory side, we need to construct more sophisticated model for these GPDs.

Lattice QCD can also study these GPDs by calculating the so-called generalized nucleon form factors. Most recent calculation [15] indicated a number of interesting features on the quark orbital angular momentum contributions from the valence quarks. First, the total spin contribution from the down quark is close to zero, which means that the quark spin contribution and orbital angular momentum contribution from the down quark tends to cancel out each other. Second, the total orbital angular momentum contribution from the up quark and down quark is close to zero too. There is a strong cancelation between the orbital angular momenta from up and down quarks. Here is one of the final results for the total quark contribution to the proton angular momentum [15],

$$
J^{u+d}=0.213(44), \quad J^{u}=0.214(27), \quad J^{d}=-0.001(27),
$$

for up and down quarks, where the numbers in the brackets are the error bars quoted in the calculation. Of course, we should also improve these calculations by including the so-called dis-connected diagrams. How this will affect the results is remained to be seen.

\section{Transverse Spin Physics}

There have been strong experimental interests on transverse spin physics around the world, from the deep inelastic scattering experiments such as the HERMES collaboration at DESY, SMC at CERN, and Hall A and CLAS at JLab, the proton-proton collider experiment from RHIC at Brookhaven, and the very relevant $e^{+} e^{-}$annihilation experiment from BELLE at KEK. One of the major goals in transverse spin physics is to study the quark transversity distribution, the last unknown leading-twist quark distribution in nucleon. We are now starting to have a first glimpse about the quark transversity distribution from the experiments (see from example [16]).

Besides the quark transversity distribution, the transverse spin physics also opened a new window to explore the partonic structure of nucleon, the so-called transverse momentum dependent 
(TMD) parton distributions [17]. TMD parton distribution is an extension to the usual Feynman parton distributions. These distributions allow us to study the three-dimension picture of partons inside the nucleon, and they are also closely related to the generalized parton distributions [18] and the parton orbital angular momenta. Especially, the single transverse spin asymmetry (SSA) phenomena in high energy hadronic processes have attracted many theoretical and experimental investigations. The SSA is defined as the asymmetry when one of the hadrons' transverse spin is flipped, $A_{N} \sim\left(d \sigma\left(S_{\perp}\right)-d \sigma\left(-S_{\perp}\right)\right) /\left(d \sigma\left(S_{\perp}\right)+d \sigma\left(-S_{\perp}\right)\right)$. It has been a theoretical challenge in the understanding of these phenomena. This is because the leading partonic contribution to the SSA vanish in the leading order, whereas the experimental observation show that these SSAs are in tens of percentage in the forward scattering of the polarized nucleon.

Recent theoretical developments have made great progress in the exploration of the underlying physics for the single spin phenomena. It is impossible to cover all these exciting physics in this short talk. Rather, I would like to focus on one important subject, i.e., the nontrivial QCD dynamics associated with transverse spin physics: the QCD factorization, and the universality of the parton distributions and fragmentation functions.

Among those TMD parton distributions and fragmentation functions, two functions have been mostly discussed: the Sivers quark distribution and the Collins fragmentation function. The Sivers quark distribution represents a distribution of unpolarized quarks in a transversely polarized nucleon, through a correlation between the quark's transverse momentum and the nucleon polarization vector. The Collins function represents a correlation between the transverse spin of the fragmenting quark and the transverse momentum of the hadron relative to the "jet axis" in the fragmentation process. Although they both belong to the so-called "naive-time-reversal-odd" functions, they do have different universality properties. For the quark Sivers function, because of the initial/final state interaction difference, they differ by signs for the SIDIS and Drell-Yan processes $[19,20,21,22]$. On the other hand, there have been several studies showing that the Collins function is universal between different processes, primarily in the SIDIS and $e^{+} e^{-}$annihilation $[23,24,25]$, and recently in $p p$ collisions [26]. In the following, I will take the example of the Collins contribution to the azimuthal asymmetric distribution of hadrons inside a high energy jet in the transversely polarized $p p$ collision to demonstrate this universality property,

$$
p\left(P_{A}, S_{\perp}\right)+p\left(P_{B}\right) \rightarrow \operatorname{jet}\left(P_{J}\right)+X \rightarrow H\left(P_{h}\right)+X,
$$

where a transversely polarized proton with momentum $P_{A}$ scatters on another proton with momentum $P_{B}$, and produces a jet with momentum $P_{J}$. The three momenta of $P_{A}, P_{B}$ and $P_{J}$ form the so-called reaction plane. Inside the produced jet, the hadrons are distributed around the jet axis, where we define transverse momentum $P_{h T}$ relative to the jet axis. The correlation between $P_{h T}$ and the polarization vector $S_{\perp}$ introduces the Collins contribution to the single spin asymmetry in this process.

We need to generate a phase from the scattering amplitudes to have a non-vanishing SSA. If the phase comes from the vertex associated with the fragmenting quark and the final state hadron, or from the dressed quark propagator, it is easy to argue the universality of the Collins function between this process and the SIDIS $/ e^{+} e^{-}$process, because they are the same. The main issue of the universality discussion concerns the extra gluon exchange contribution between the spectator of the fragmentation process and hard partonic part. In Fig. 2, we have shown all these interactions 


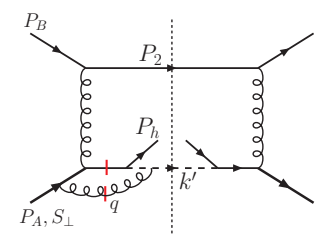

(a)

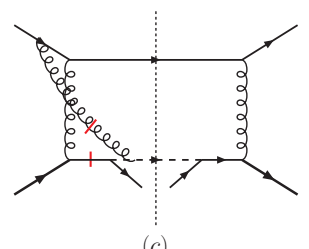

(c)

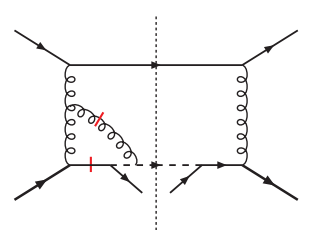

$(b)$

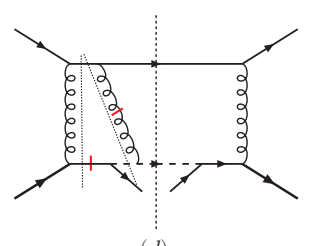

$(d)$

Figure 1: Gluon exchange diagrams contributions to the Collins asymmetry in $p p$ collisions. The short bars indicate the pole contributions to the phase needed for a non-vanishing SSA. The additional two cuts in (d) cancel out each other.

for a particular partonic channel $q q^{\prime} \rightarrow q q^{\prime}$ contribution, including the gluon attachments to the incident quarks (a,c), and final state balancing quark (d) and the internal gluon propagator (b). The contributing phases of the diagrams in Fig. 2 come from the cuts through the internal propagators in the partonic scattering amplitudes. In Fig. 2, we labeled these cut-poles by short bars in the diagrams. From the calculations, we will find that all these poles come from a cut through the exchanged gluon and the fragmenting quark in each diagram, and all other contributions either vanish or cancel out each other. For example, in Fig. 2(d), we show two additional cuts, which contribute however opposite to each other and cancel out completely. Therefore, by using the Ward identity at this particular order, the final results for all these diagrams will sum up together into a factorized form, where the cross section is written as the hard partonic cross section for $q\left(S_{\perp}\right) q^{\prime} \rightarrow q\left(s_{\perp}\right) q^{\prime}$ subprocess multiplied by a Collins fragmentation function. The exchanged gluon in Fig. 2 is now attaching to a gauge link from the fragmentation function definition. Similar calculations can be performed for the other two processes SIDIS and $e^{+} e^{-}$annihilation, and the same Collins function will be observed. This argument can also be extended to two-gluon exchange diagrams [26].

The key steps in the above derivation are the eikonal approximation and the Ward identity. The eikonal approximation is valid when we calculate the leading power contributions in the limit of $P_{h T} \ll P_{J}$. The Ward identity ensure that when we sum up the diagrams with all possible gluon attachments we shall get the eikonal propagator from the gauge link in the definition of the fragmentation function. The most important point to apply the Ward identity in the above analysis is that the eikonal propagator does not contribute to the phase needed to generate a nonzero SSA.

This observation is very different from the SSAs associated with the parton distributions, where the eikonal propagators from the gauge link in the parton distribution definition play very important role $[17,19,20,21,22]$. It is the pole of these eikonal propagators that contribute to the phase needed for a nonzero SSA associated with the naive-time-reversal-odd parton distributions, which also predicts a sign difference for the quark Sivers function between the SIDIS and Drell-Yan processes. More complicated results have been found for the SSAs in the hadronic dijetcorrelation [27, 28], where a normal TMD factorization breaks down [29]. The reason is that the 
eikonal propagators from the initial and final state interactions in dijet-correlation process do contribute poles in the cross section [28, 29]. Because of this, the Ward identity is not applicable, and the standard TMD factorization breaks down, although a modified factorization may be valid if we modify the definition of the TMD parton distributions to take into account all the initial and final state interaction effects [27].

As mentioned above, there exists a nontrivial relation between the SSAs in SIDIS and DrellYan processes [19, 20],

$$
\text { Sivers SSA }\left.\right|_{\text {DY }}=- \text { Sivers SSA }\left.\right|_{\text {DIS }} \text {. }
$$

More importantly, the opposite sign between the above two processes will still hold when gluon radiation contributions are taken into account, where the large transverse momentum Sivers function is generated from the twist-three quark-gluon correlation function [30, 31]. It is of crucial importance to test this nontrivial QCD predictions by comparing the SSAs in these two processes. The Sivers single spin asymmetry in SIDIS process has been observed by the HERMES collaboration, and the planned Drell-Yan measurement at RHIC and other facility will test this prediction.

Another interesting probe for the initial/final state interaction effects is the SSA in heavy quark and antiquark production in hadronic process. Because the heavy quark and antiquark can be detected by their decay products, their SSAs can be measured separately. The heavy quark and antiquark produced in short distance partonic processes will experience different final state interactions with the nucleon spectator due to their different color charges, and therefore the SSAs for heavy quark and antiquark will be different. Detailed calculations show that the difference could be as large as a factor of 3 if the quark-antiquark channel contribution dominates [32].

In summary, the universality of the parton distribution and fragmentation functions are very different in the single transverse spin asymmetry. These properties are still under theoretical and experimental investigations. These important physics, together with other exciting features have shown that the transverse spin physics is playing a very important role in the strong interaction physics for hadronic spin physics. We will learn more about QCD dynamics and nucleon structure from these studies.

This work was supported in part by the U.S. Department of Energy under grant contract DEAC02-05CH11231. We thank RIKEN, Brookhaven National Laboratory and the U.S. Department of Energy (contract number DE-AC02-98CH10886) for providing the facilities essential for the completion of their work.

\section{References}

[1] J. Ashman et al. [European Muon Collaboration], Nucl. Phys. B 328, 1 (1989).

[2] B. W. Filippone and X. D. Ji, Adv. Nucl. Phys. 26, 1 (2001), and references therein.

[3] A. Airapetian et al. [HERMES Collaboration], Phys. Rev. D 75, 012007 (2007).

[4] V. Y. Alexakhin et al. [COMPASS Collaboration], Phys. Lett. B 647, 8 (2007).

[5] G. R. Farrar, D. R. Jackson, Phys. Rev. Lett. 35, 1416 (1975).

[6] X. Zheng et al., Phys. Rev. Lett. 92, 012004 (2004); Phys. Rev. C 70, 065207 (2004).

[7] H. Avakian, S. J. Brodsky, A. Deur and F. Yuan, Phys. Rev. Lett. 99, 082001 (2007). 
[8] H. Jackson, for the HERMES collaboration, talk given at the DIS 08 workshop, London, April 7-12, 2008.

[9] A. Adare et al. [PHENIX Collaboration], Phys. Rev. D 76, 051106 (2007).

[10] B. I. Abelev et al. [STAR Collaboration], Phys. Rev. Lett. 100, 232003 (2008).

[11] D. de Florian, R. Sassot, M. Stratmann and W. Vogelsang, Phys. Rev. Lett. 101, 072001 (2008).

[12] X. Ji, Phys. Rev. Lett. 78, 610 (1997).

[13] M. Mazouz et al. [Jefferson Lab Hall A Collaboration], Phys. Rev. Lett. 99, 242501 (2007).

[14] A. Airapetian et al. [HERMES Collaboration], JHEP 0806, 066 (2008).

[15] Ph. Hagler et al. [LHPC Collaborations], Phys. Rev. D 77, 094502 (2008).

[16] M. Anselmino, M. Boglione, U. D’Alesio, A. Kotzinian, F. Murgia, A. Prokudin and C. Turk, Phys. Rev. D 75, 054032 (2007).

[17] P .Mulders, these proceedings.

[18] B. Pasquini, these proceedings.

[19] S. J. Brodsky, D. S. Hwang and I. Schmidt, Phys. Lett. B 530, 99 (2002); Nucl. Phys. B 642, 344 (2002).

[20] J. C. Collins, Phys. Lett. B 536, 43 (2002).

[21] X. Ji and F. Yuan, Phys. Lett. B 543, 66 (2002); A. V. Belitsky, X. Ji and F. Yuan, Nucl. Phys. B 656, 165 (2003).

[22] D. Boer, P. J. Mulders and F. Pijlman, Nucl. Phys. B 667, 201 (2003).

[23] A. Metz, Phys. Lett. B 549, 139 (2002).

[24] J. C. Collins and A. Metz, Phys. Rev. Lett. 93, 252001 (2004).

[25] L. P. Gamberg, A. Mukherjee and P. J. Mulders, Phys. Rev. D 77, 114026 (2008).

[26] F. Yuan, Phys. Rev. Lett. 100, 032003 (2008); Phys. Rev. D 77, 074019 (2008).

[27] C. J. Bomhof, P. J. Mulders and F. Pijlman, Phys. Lett. B 596, 277 (2004); Eur. Phys. J. C 47, 147 (2006); JHEP 0702, 029 (2007); A. Bacchetta, C. J. Bomhof, P. J. Mulders and F. Pijlman, Phys. Rev. $D$ 72, 034030 (2005); C. J. Bomhof and P. J. Mulders, arXiv:0709.1390 [hep-ph].

[28] J. W. Qiu, W. Vogelsang and F. Yuan, Phys. Lett. B 650, 373 (2007); Phys. Rev. D 76, 074029 (2007); W. Vogelsang and F. Yuan, Phys. Rev. D 76, 094013 (2007).

[29] J. Collins and J. W. Qiu, Phys. Rev. D 75, 114014 (2007); J. Collins, arXiv:0708.4410 [hep-ph].

[30] X. Ji, J. W. Qiu, W. Vogelsang and F. Yuan, Phys. Rev. Lett. 97, 082002 (2006); Phys. Rev. D 73, 094017 (2006); Phys. Lett. B 638, 178 (2006).

[31] M. Diehl, these proceedings.

[32] F. Yuan and J. Zhou, Phys. Lett. B 668, 216 (2008). 